\title{
Amplitude Regeneration of Phase Encoded Signals Using Injection Locking in Semiconductor Lasers
}

\author{
A. Fragkos ${ }^{1}$, A. Bogris ${ }^{2}$ and D. Syvridis ${ }^{1}$, R.Phelan ${ }^{3}$, J. O'Carroll ${ }^{3}$, B.Kelly ${ }^{3}$ and J.O'Gorman ${ }^{3}$ \\ 1) Optical Communications Laboratory, Department of Informatics and Telecommunications, University of Athens, Panepistimiopolis, Illisia, \\ Athens, GR-15784, Greece \\ 2) Department of Informatics, Technological Educational Institute of Athens, Aghiou Spiridonos, 12210 Egaleo, Athens, Greece \\ 3) Eblana Photonics, Trinity College Enterprise Centre, Pearse Street, Dublin 2, Ireland \\ e-mail:alx_f@di.uoa.gr
}

\begin{abstract}
A phase preserving limiter based on injection locking in semiconductor lasers is experimentally investigated for $10 \mathrm{~Gb} / \mathrm{s}$ phase encoded signals. The proposed scheme exhibits significant amplitude noise squeezing in conjunction with extreme simplicity.

OCIS codes: (140.0140) Lasers and laser optics; (140.3520) Lasers, injection-locked
\end{abstract}

\section{Introduction}

The constantly increasing need for higher bit rates and longer optical transmission paths has led to alternative, more resilient to fiber losses and bandwidth efficient, modulation formats such as Phase Shift Keying (PSK). Phase modulated signals exhibit a higher receiver sensitivity over conventional On-Off Keying (OOK) systems and suitability for multi-level signaling. The major threat for phase modulation formats is the non-linear phase noise, which is produced by the accumulated amplitude noise generated by optical amplifiers and transferred to signal phase through the self-phase modulation (SPM) and cross-phase modulation (XPM) nonlinear effects. A very efficient way to reduce the non-linear phase noise is to suppress the amplitude noise of the degraded signal somewhere in the middle of the transmission link or in a periodic manner. Amplitude limiters based on Non-Linear Amplifying Loop Mirror (NALM) [1], and on saturated four wave mixing (FWM) [2] in highly nonlinear optical fibers are the most prominent schemes. Both techniques have been proven to exhibit significant noise suppression features, however their use in a commercial transmission system is still not attractive due to their increased size and the need for extra components such as optical amplifiers and optical filters. In this paper we propose an alternative amplitude limiter based on optical injection locking in a discrete mode semiconductor laser [3] that offers enhanced amplitude noise squeezing, implementation simplicity and ability to reproduce the phase modulation bandwidth of the incoming signal up to multi $\mathrm{GHz}$ bandwidth, thus enabling the amplitude regeneration of constant envelope phase encoded signals. The experiment demonstrates the regeneration principle for $10 \mathrm{~Gb} / \mathrm{s}$ differential PSK (DPSK) signals. The technique could be extended for M-PSK signals as well.

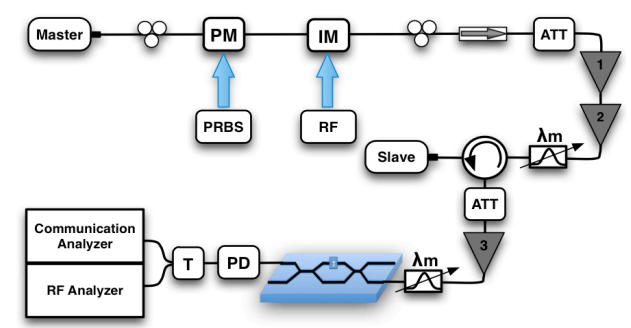

Fig. 1. Block diagram of the experimental set-up. PM: Phase Modulator, IM: Intensity Modulator, PRBS: Pseudo Random Binary Sequence, RF: Radio Frequency Wave Generator, EDFA1: OSNR Controller, EDFA2: Injection Level Controller, EDFA3: Receiver Amplifier, PD: Photodiode and T: RF Splitter.

\section{Experimental results and discussion}

Up to date, injection locking was utilized for the enhancement of linewidth and intensity noise characteristics of semiconductor lasers [4]. In this paper, we show that injection locking is not only a regenerative amplifier of optical carriers [4, 5], but of carrier-suppressed phase encoded signals as well. Since the scheme is tested for $10 \mathrm{~Gb} / \mathrm{s} \mathrm{PSK}$ signals, the slave laser current must be tuned at values which reassure that its intrinsic modulation bandwidth is of the same order. The second parameter that determines the locking bandwidth is the power of the master injected into the slave. The injection level must be properly adjusted so as to enable the reproduction of master phase modulation 


\section{OWG1.pdf}

at the slave with the minimum transfer of its amplitude noise properties. The block diagram of the experimental setup is depicted in figure 1. A tunable external cavity laser is used as the master laser and is phase modulated at 10 $\mathrm{Gb} / \mathrm{s}$ with the use of a phase modulator and a pseudo random binary sequence (PRBS) generator. The bit sequence is a $2^{31}-1$ one. The polarization controller before the phase modulator is used in order to align the laser with the phase modulation axis of the modulator. The variable attenuator before the first erbium doped fiber amplifier (EDFA) controls the optical signal to noise ratio (OSNR) of the transmitted PSK signal while the second EDFA controls the injection level of the signal that is injected into the slave laser. The output of the second EDFA is filtered in order to remove amplified spontaneous emission noise (ASE) with the use of a band-pass filter of $1 \mathrm{~nm}$ bandwidth and then is fed into the slave laser via a circulator. The single mode slave laser exhibits $10 \mathrm{~Gb} / \mathrm{s}$ modulation bandwidth at currents close to $98 \mathrm{~mA}$. The output of the slave laser is sent to a second variable attenuator and then amplified by a third EDFA which plays the role of the pre-amplifier before the receiver required for systematic bit-error rate (BER) measurements. The output of the master is also launched into the same amplifier in order to provide a fair comparison of the master and slave BER potential. Finally, both slave and master outputs are coupled into a $100 \mathrm{ps}$ delay interferometer for the differential detection at a single $12 \mathrm{GHz}$ bandwidth photodiode and monitored in a 20 $\mathrm{GHz}$ communication analyzer and a $26 \mathrm{GHz}$ spectrum analyzer. The experimental study is carried out for two noise regimes. First, we investigate the performance of the regenerator as an inline amplitude limiter where ASE noise of the EDFA affects both amplitude and phase of the signal. Then we evaluate the regenerative characteristics of injection locking in a special case where the signal is only impaired by amplitude noise. This noise regime seems artificial; however noise only at the amplitude could appear at the output of a phase sensitive amplifier-regenerator (PSA) operating in the linear regime [5]. It is known, that a linear PSA squeezes perfectly the phase noise of phasemodulated signals, however the phase noise is converted to amplitude noise through the phase sensitive amplification process.

First we investigated the case where the master is degraded by ASE noise. For different noise levels of the master, we investigated the amplitude squeezing properties of the injection locked laser (fig. 2).

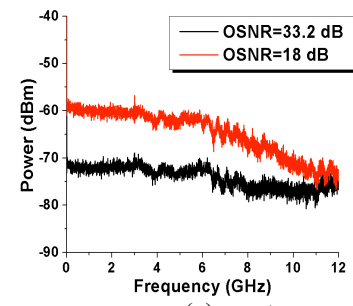

(a) master

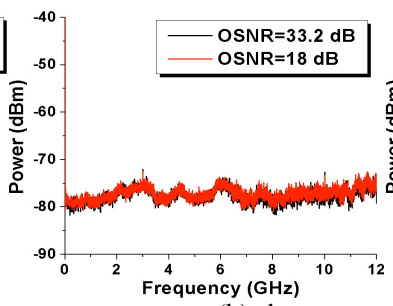

(b) slave

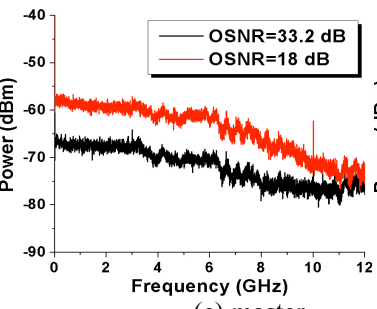

(c) master

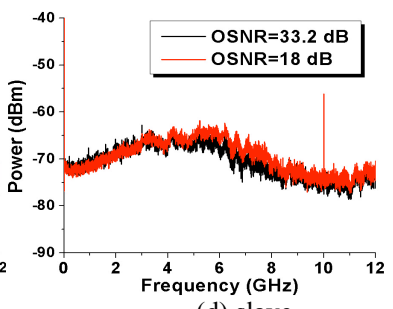

(d) slave

Fig.2. Master and slave power spectral density, before the delay interferometer, without (a, b) and with (c, d) $10 \mathrm{~Gb} / \mathrm{s}$ phase modulation at master for two master OSNR values.

In fig. 2 we notice that for typical carriers, the slave laser presents a constant SNR regardless of the master laser noise properties (fig.2 (a, b)). When a $10 \mathrm{~Gb} / \mathrm{s}$ phase-modulated signal is the input of the slave, the slave remains unaffected by master noise, but also presents a swelling of the noise spectral density around $4 \mathrm{GHz}$ compared to the case of no modulation. This effect is the result of the conversion of $10 \mathrm{~Gb} / \mathrm{s}$ phase modulation to amplitude fluctuations inside the slave and its origin should be thoroughly explored towards the optimization of the limiting amplifier. Despite this side effect, the noise integration over $10 \mathrm{GHz}$ bandwidth provides a smaller noise power for the slave when master OSNR is equal to $18 \mathrm{~dB}$ especially close to lower frequencies. The total noise suppression for the specific OSNR turns out to be $7 \mathrm{~dB}$. To evaluate the regenerative performance of the limiter we performed BER measurements as a function of the receiver power for a constant OSNR $=25 \mathrm{~dB}$ and as a function of master OSNR for a receiver power equal to $P_{\text {rec }}=-7.5 \mathrm{dBm}$. The results are promising as they reveal that the injection locked laser provides a significant power penalty improvement of $1.5 \mathrm{~dB}$ (fig.3, left diagram) and relaxes the required OSNR for a given BER by $1.5 \mathrm{~dB}$ as well. It must be noted that injection locking operates as an amplifier in these experiments. Its gain was measured to be close to $20 \mathrm{~dB}$.

In the second case, we considered severe noise degradation only at the intensity of the master trying to emulate the case where phase noise is removed by a linear phase sensitive regenerator [5]. The experimental analysis that follows shows that an injection locked semiconductor laser could comprise the ideal regenerator to handle intensity noise degraded phase encoded signals. To experimentally emulate the intensity noise case, we applied sinusoidal perturbation to the NRZ-DPSK signal with the use of an intensity modulator. 


\section{OWG1.pdf}
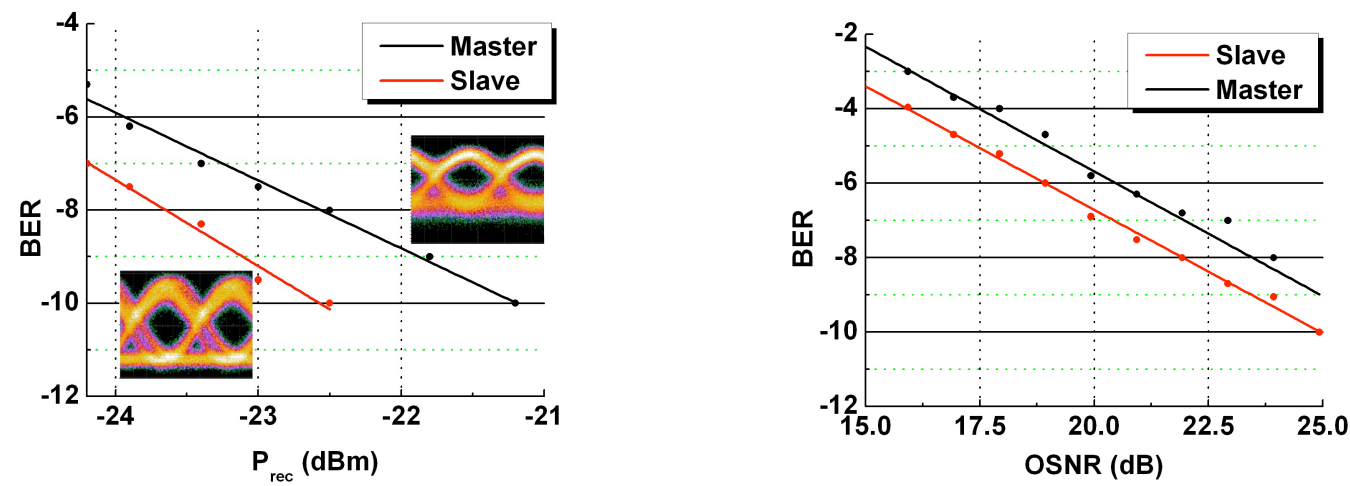

Fig.3. (Left diagram) BER measurement as a function of the receiver power for master OSNR equal to $30 \mathrm{~dB}$. (Right diagram) BER is a function of the master OSNR keeping the received power equal to $-7.5 \mathrm{dBm}$

The results show remarkable regenerative performance. Error free operation $\left(\mathrm{BER}<10^{-9}\right)$ is recorded for the output of the slave $\left(\mathrm{P}_{\text {rec }}=-15 \mathrm{dBm}\right)$ whilst the master BER is limited to $10^{-5}$. The eye-diagrams reveal the perfect squeezing of the amplitude perturbation after injection locking. It must be noted that similar squeezing performance was recorded for all frequencies from 1 to $10 \mathrm{GHz}$.

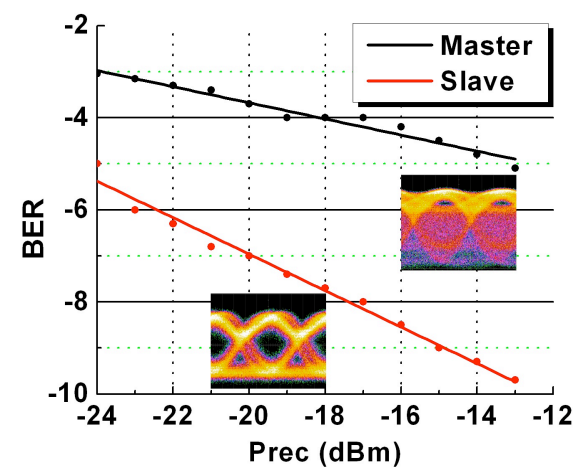

Fig.4. BER measurement as a function of the receiver power when the master is perturbed by a strong $1 \mathrm{GHz}$ sinusoidal signal, (master OSNR $=30 \mathrm{~dB}$ )

\section{Conclusion}

An alternative amplitude limiter based on injection locking in semiconductor lasers has been proposed and experimentally studied for $10 \mathrm{~Gb} / \mathrm{s}$ PSK signals. The experiments showed efficient amplitude noise squeezing and improvement of the power penalty by $1.5 \mathrm{~dB}$. The limiter showed even better performance for signals solely impaired at the amplitude, emerging as a potential amplitude equalizer after a phase sensitive amplifier. The scheme could operate up to 25 Gbaud using commercially available sources. The great efficiency of the regenerator combined with the simplicity and the potential for monolithic integration even for multi-channel (multi-laser device) regeneration make it a promising candidate for use in transmission systems of $100 \mathrm{~Gb} / \mathrm{s}$ Ethernet.

\section{Acknowledgement}

We would like to thank Phoenix Photonics for the donation of the delay interferometer. This work was funded by the European Commission STREP project PHASORS (FP7-ICT-2007-2 22457), the Iraklitos grant of Hellenic Ministry of Education and 216863 (BONE).

\section{References}

[1] C. Stephan et. Al., "Nonlinear Phase Noise Reduction in a DPSK Transmission System Using Cascaded Nonlinear Amplifying Loop Mirrors," IEEE Photon. Technol. Lett. 21, 1864-1866 (2009).

[2] M. Matsumoto and K. Sanuki, "Performance improvement of DPSK signal transmission by a phase-preserving amplitude limiter," Opt. Express. 15, 8094-8103 (2007).

[3] R. Phelan, B. Kelly, J. O'Carroll, C. Herbert, A. Duke and J. O'Gorman. "Room-Temperature Operation of Discrete-Mode InGaAs-AlAsSb Quantum-Cascade Laser With Emission at $\lambda=3.3 \mathrm{~nm}$," IEEE Electronics Letters, 45, 43-45, (2009)

[4] Akira Furusawa, "Amplitude squeezing of a semiconductor laser with light injection," Opt. Lett. 21, 2014-2016, (1996)

[5] R. Slavik, et al, "All-optical phase and amplitude regenerator for next-generation telecommunications systems," Nature Photonics, 4, 690695, (2010) 